\title{
RELATIONSHIP BETWEEN BLOOD PRESURE, CEREBRAL BLOOD FLOW VELOCITY AND WHITE MATTER LESIONS IN PATIENTS WITH HYPERTENSIVE ENCEPHALOPATHY
}

\author{
S. Andonova, D. Minchev \\ Medical University of Varna, Department of Neurology \\ Reviewed by: Assoc. prof. L. Havezova, PhD
}

\begin{abstract}
Purpose: The aim of this study is to find the relationships between blood pressure, cerebral blood flow velocity and white matter lesions. The blood pressure lowering could slow the progression of disease. Methods: By 23 elderly individuals (mean age $\mathbf{- 6 5 , 7}$ years) with hypertensive encephalopathy was measured a blood flow velocity (BFV) and cerebral vasomotor reactivity(CVR) in the middle cerebral artery using a 2 -MHz pulsed Transcranial Doppler. MRI was done at the beginning of the study and at 24 months to measure the severity of white matter hypertensities. The blood pressure was measurent frecuently and on these basis the patients were divided into two groups: A-9 patients with hight hypertension and B-14 patients with low or middle. All used an ACE-inhibitor alone or in combination with a diuretic. Results: The differences in blood flow velocity and cerebral vasomotor reactivity and MRI findings- hyperintensity periventricular and in deep white matter were significant among different severity of blood pressure. Group A - mean BFV-left $40+/-10 \mathrm{sm} / \mathrm{s}$; right-42 +/- $10 \mathrm{sm} / \mathrm{s} ;$ IR-0,65-0,68 Group B - mean BFV-left 54+/- $10 \mathrm{sm} / \mathrm{s} ;$ right-56 +/- $10 \mathrm{sm} / \mathrm{s}$, IR-0,60-0,64 $(\mathbf{p}<0,001)(f i g$ 1).Elevated hypertension correlated with grow of white matter hypertensities on MRI. Conclusions: The findings in our study suggest that dysfunction of cerebral blood flow velocity might be an important factor in the development of white matter lesions in elderly with hypertension. The treatment with antihypertensive agents lower risk of developing new white matter hypertensities and slowed the progression of hypertensive encephalopathy.
\end{abstract}

The aim of the study is to find the relationships between blood pressure, cerebral blood flow velocity and white matter lesions on MRI. The blood pressure lowering could slow the progression of disease.

Hypertensive arteriopathy: The microangiopathy of arterial hypertension has been studied. The main lesions are microatheromata, lipohyalinosis and fibrinoid necrosis. Minute foci of microatheromatosis produse stenosis or occlusion of arterioles in hypertensive individuals. Lipohyalinosis is a progressive disorganisation of small arthery walls with subependimal deposits of a hyaline fibrinoid substance. Lipohyalinosis leads either to thrombotic occlusion of the lumen and lacunar stroke or to mural destruction with formation of microaneurysms and hypertensive cerebral haemorrhage. Fibrinoid angionecrosis occurs with extreme hypertension produsing segmental narrowing, dilatation and necrosis of the vessel wall with deposits of a brightly eosinophilic substance. The perivascular tissues around the constricted spastic segments are destroyed and astrocytic oedema is present. $(1,2,3)$

White matter lesions: Vascular lesions of the cerebral white matter are best visualized in MRI. Extensive white

Address for correspondence:

S. Andonova, Dept. of Neurology, Medical University Prof. Dr.

Paraskev Stoyanov, 55 Marin Drinov St., BG-9002, Varna, BULGARIA matter lesions can also occur in cerebral amyloid angiopathy. Collagenous thickening and occlusion of deep periventricular -draining veins has been postulated to be another factor in the production of deep hemispheric white matter lesions. Subependimal lesions in the immediate vicinity of the ventricles correspond to decreased myelin, loss ependymal cells, reactive gliosis, and increased extracellular fluid. Subependymal lesions can be found at any age and are probably non pathological. $(4,7)$

Pathophysiology: The pathophysiological mechanisms include haemorheological factors, increased resistance to flow,decreased autoregulation, endothelial changes,dysfunction of the blood-brain barrier and dilatation of perivascular spaces.Their combined effects result in hypoperfusion and incomplete infarction of deep white matter. $(5,6)$

Incomplete infarction; Below a critical perfusion thereshold, selective cell loss may occur without pronounced infarction or cystic necrosis.Selective neuronal loss occurs in the penumbra surrounding acute infarcts, whereas selective loss of oligodendrocytes,myelin and axons occurs in deep white matter of patients with severe stenosis of medullary arterioles. The selective loss of tissue elements due to ischaemia is known as incomplete infarction. And may occur when systemic blood pressure drops below autoregulatory reserve,intracranial pressure 
exceed maen arterial pressure or there is severe stenosis of several arteries or arterioles. $(1,2)$

In the population-based Cardiovascular Health Study,about a quarter of 3660 participans aged 650 r older had one or more lacunes on MRI. Most lacunes (89\%) were clinically silent. In other population- based studies the prevalence of silent lacunes ranged from $11 \%$ to $24 \%$.Incomplete or non cavitating ischaemic white -matter lesions of the brain -with or without lacunes decrease in elderly people.The prevalence of cerebral white -matter hyperintensities on MRI in elderly with hypertension is in the range of $62-95 \%$.These lesions are associated with advancing age,heart disease,smoking. Cognitiv disfunction and gait impairment are related to lesion sevsrity.White -matter lesions are seen as bilaterally symmetrical areas of hypodensity on CT and as hyperintensities in the periventricular or deep subcortical white matter on T2 -weighted MRI.

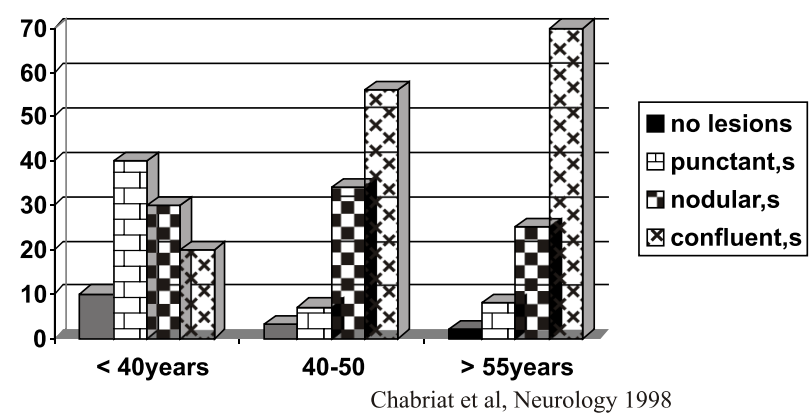

Fig. 1. Scale with the degree of white matter lesions, depend from age:

In our study -by 23 individuals (mean age 65,7 years) with hypertensive encephalopathy was measured a blood flow velocity (BFV) and cerebral vasomotor reactivity (CVR) in the middle cerebral artery using a 2-MHz pulsed Transcranial Doppler. MRI was done at the beginning of the study and at 24 months to mesure the severity of white matter hyperintensities. The blood pressure was measurent frecuently and on these basis the patients were divided into two groups: A-9 patients with high hypertension and B-14 patients with low or middle. All used an ACE-inhibitor alone or in combination with a diuretic.
The differences in blood flow velocity and cerebral vasomotor reactivity and MRI findings -hyperintensity peruventricular and in deep white matter were significant among different severity of blood pressure. Group A -mean BFV -Left $40+/-10 \mathrm{sm} / \mathrm{s}$; right- $42+/ 10 \mathrm{sm} / \mathrm{s} ; \mathrm{IR}-$ 0,65-0,68; Group B-mean BFV-left- $54+/-10 \mathrm{sm} / \mathrm{s}$; right$56+/-10 \mathrm{sm} / \mathrm{s}$; IR- 0,60-0,64; ( $<<0,001)$ (fig 1). Elevated hypertension correlated with grow of white matter hypertensities on MRI.

The findings in our study suggest that dysfunction of cerebral blood flow velocity might be an important factor in the development of white matter lesions in elderly with hypertension. The treatment with antihypertensive agents lower risk of developing new white matter hypertensities and slowed the progression of hypertensive encephalopathy.

\section{REFERENCES}

1. Roman G, Wallin A., Chui H.,Subcortical ischaemic vascular dementia, The Lancet, Neurology, vol 1 2002,426-434

2. Kinkel W., Jacobs L.,Polachini I., Subcortical arteriosclerotic encephalopathy, Neurology, vol 42, 10,1985

3. Inzitari M, Leukoaraiosis: An independent risk factor for stroke, Stroke 2003, 34, 2067-2071

4. Fukuda, Kitani, Differences between treated and untreated hypertensive subjects in the extent of periventricular hyperintensities observed on brain MRI, Stroke 1995,26,1593-1597

5. Roman GC, Tatemichi TK, Erkinjuntti T. Vascular dementia. Diagnostic criteria for research studies. Report of the NIDS AIREN International Workshop. Neurology 1993, 43,250 60.

6. Roman CG. Cognitive Decline and Vascular Dementia The Silent Epidemic of the 21 st Century, Neuroepidemiology, 2003, 22, 161-164.

7. Schwarz RB. Jones KM. Mantello MT: Hypertensive encephalopathy :findings on CT,MR imaging;AJR Am J Roentgenol;1992 Aug;159\2\ 